\title{
mRNA COVID-19 vaccines induce enhanced antibody and cellular responses compared to ChAd0x1 or natural infection in children
}

Alexander Dowell ( A.C.Dowell@bham.ac.uk)

University of Birmingham https://orcid.org/0000-0002-1909-7047

Annable Powell

UK Health Security Agency

Chris Davis

University of Glasgow

Brian Willett

MRC-University of Glasgow Centre for Virus Research https://orcid.org/0000-0001-8912-3266

Rachel Bruton

University of Birmingham

Morenike Ayodele

University of Birmingham

Elizabeth Jinks

University of Birmingham

Panagiota Sylla

University of Birmingham

Eliska Spalkova

University of Birmingham

Samantha Nicol

University of Birmingham

Jianmin Zuo

University of Birmingham https://orcid.org/0000-0002-8341-465X

Georgina Ireland

Public Health England https://orcid.org/0000-0001-8932-2783

Ifeanyichukwu OKIKE

UK Health Security Agency

Frances Baawuah

Public Health England

Joanne BECKMANN

East London NHS Foundation Trust

Shazaad Ahmad 
Manchester University NHS Foundation Trust

Joanna GARSTANG

Birmingham Community Healthcare NHS Trust

Andrew Brent

Oxford University Hospitals NHS Trust

\section{Bernadette Brent}

Oxford University Hospitals NHS Foundation Trust

\section{marie white}

Evelina London Children's Hospital

\section{Aedin Collins}

Tallaght University Hospital

\section{Fracesca Davis}

Evelina London Children's Hospital

ming lim

https://orcid.org/0000-0001-7738-8910

\section{Julia Kenny}

Guy's and St Thomas' NHS Foundation Trust

\section{Jonathan Cohen}

Evelina London Children's Hospital https://orcid.org/0000-0003-1004-5598

\section{Mary Ramsay}

Public Health England

\section{Ezra Linley}

Public Health England

\section{John Poe}

UK Health Security Agency

\section{Gayatri Amirthalingam}

UK Health Security Agency https://orcid.org/0000-0003-2078-0975

\section{Kevin Brown}

Public Health England https://orcid.org/0000-0002-1568-683X

\section{Rafaq Azad}

Bradford Teaching Hospitals NHS Foundation Trust

\section{Dagmar Waiblinger}

Bradford Teaching Hospitals NHS Foundation Trust

\section{John Wright}

Bradford Teaching Hospitals NHS Trust

\section{Paul Moss}

University of Birmingham https://orcid.org/0000-0002-6895-1967

\section{Shamez Ladhani}

Public Health England 


\section{Brief Communication}

Keywords: mRNA COVID-19 vaccines, antibody and cellular responses, COVID-19

Posted Date: November 24th, 2021

DOI: https://doi.org/10.21203/rs.3.rs-1108654/v1

License: (c) (i) This work is licensed under a Creative Commons Attribution 4.0 International License. Read Full License 


\section{mRNA COVID-19 vaccines induce enhanced antibody and cellular responses compared to ChAdOx1 or natural infection in children}

Alexander C. Dowell ${ }^{1 *}$, Annabel A. Powell ${ }^{2 *}$, Chris Davis ${ }^{3}$, Brian J. Willet ${ }^{3}$, Rachel Bruton ${ }^{1}$, Morenike Ayodele ${ }^{1}$, Elizabeth Jinks ${ }^{1}$, Eliska Spalkova ${ }^{1}$, Panagiota Sylla ${ }^{1}$, Samantha M. Nicol ${ }^{1}$, Jianmin Zuo ${ }^{1}$, Georgina Ireland ${ }^{2}$, Ifeanyichukwu Okike ${ }^{2,4}$, Frances Baawuah ${ }^{4}$, Joanne Beckmann ${ }^{5}$, Shazaad Ahmad ${ }^{6}$, Joanna Garstang ${ }^{7}$, Andrew J Brent ${ }^{8,9}$, Bernadette Brent ${ }^{8}$, Marie White ${ }^{10}$, Aedin Collins ${ }^{11}$, Francesca Davis $^{10}$, Ming Lim ${ }^{12,13}$, Julia Kenny ${ }^{13,14}$, Ezra Linley ${ }^{15}$, John Poh ${ }^{2}$, Gayatri Amirthalingam², Kevin Brown ${ }^{2}$, Mary E Ramsay ${ }^{2}$, Rafaq Azad ${ }^{16}$, John Wright ${ }^{16}$, Dagmar Waiblinger ${ }^{16}$, Paul Moss ${ }^{1 *}$, Shamez N. Ladhani ${ }^{2,17 *}$

*These authors contributed equally

1. Institute of Immunology \& Immunotherapy, College of Medical and Dental Sciences, University of Birmingham, Birmingham, B15 2TT, UK

2. UK Health Security Agency, 61 Colindale Avenue, London NW9 5EQ, UK

3. MRC-University of Glasgow Centre for Virus Research, 464 Bearsden Road, Glasgow G61-1QH, UK

4. University Hospitals of Derby and Burton NHS Foundation Trust, Uttoxeter New Road, Derby DE22 3NE, UK

5. East London NHS Foundation Trust, 9 Allie Street, London E1 8DE, UK

6. Manchester University NHS Foundation Trust, Oxford Road, Manchester M13 9WL, UK

7. Birmingham Community Healthcare NHS Trust, Holt Street, Aston B7 4BN, UK

8. Oxford University Hospitals NHS Foundation Trust, Old Road, Oxford OX3 7HE

9. University of Oxford, Wellington Square, Oxford OX1 2JD, UK

10. Department of General Paediatrics, Evelina London Children's Hospital, London, SE1 7EH, UK

11. Tallaght University Hospital, Dublin, D24 NR0A, Ireland

12. Children's Neurosciences, Evelina London Children's Hospital at Guy's and St Thomas' NHS Foundation Trust, King's Health Partners Academic Health Science Centre, London, UK.

13. Department Women and Children's Health, School of Life Course Sciences (SoLCS), King's College London, UK

14. Department of Paediatric Infectious diseases and Immunology, Evelina London

15. UK Health Security Agency, Manchester Royal Infirmary, Manchester, UK

16. Bradford Institute for Health Research, Bradford Teaching Hospitals NHS Foundation Trust, Bradford, BD9 6RJ, UK.

17. Paediatric Infectious Diseases Research Group, St. George's University of London, London, UK 


\section{Abstract}

We present a comprehensive analysis of antibody and cellular responses in children aged 12-16 years who received COVID-19 vaccination with ChAdOx1 $(n=6)$ or mRNA vaccine (mRNA-1273 or BNT162b2, $n=9$ ) using a 12-week extended-interval schedule. mRNA vaccination of seropositive children induces high antibody levels, with one dose, but a second dose is required in infection-naïve children. Following a second ChAdOx1 dose, antibody titres were higher than natural infection, but lower than mRNA vaccination. Vaccination induced live virus neutralising antibodies against Alpha, Beta and Delta variants, however, a second dose is required in infection-naïve children. We found higher T-cell responses following mRNA vaccination than ChAdOx1. Phenotyping of responses showed predominantly early effector-memory CD4 T cell populations, with a type-1 cytotoxic cytokine signature, with IL-10. These data demonstrate mRNA vaccination induces a co-ordinated superior antibody and robust cellular responses in children. Seronegative children require a prime-boost regime for optimal protection. 
SARS-CoV-2 infection in children and adolescents is generally mild, transient and self-limiting ${ }^{1}$. However, infection results in lost time in education and children may play a role in the spread of SARSCoV-2. Children, however, may develop the inflammatory condition Multisystem inflammatory syndrome in children (MIS-C) following infection ${ }^{1}$, and those with underlying co-morbidities, in particular, may develop severe COVID-19 ${ }^{1}$. As such, vaccination of children against COVID-19 is of considerable interest.

COVID-19 vaccines were first licensed for adults in December 2020 and have proven to be highly effective. Consequently, in the United Kingdom, the Joint Committee on Vaccination and Immunisation (JCVI) recommended COVID-19 vaccination for older children aged $\geq 12$ years with severe neuro-disabilities, who are at higher risk of severe COVID ${ }^{1}$. This recommendation was made in December 2020 at the same time as recommendations for adults with underlying comorbidities, although COVID-19 vaccines were not approved for children at that time. Both Moderna (mRNA1273), and Pfizer-BioNTech (BNT162b2) mRNA vaccines and the AstraZeneca adenoviral-vector (ChAdOx1) vaccine were recommended for children with severe neuro-disabilities.

Subsequent studies have indicated mRNA vaccination of children is highly protective ${ }^{2,3}$ and durable ${ }^{4}$. However, the underlying immune response generated has yet to be fully understood. Currently, there are also no comparative data on the relative immunogenicity of adenoviral vector vaccines compared to mRNA vaccines in children. Whilst, we have previously shown children aged 3-11 years mount robust, durable, spike-dominated cellular and antibody response to natural-infection ${ }^{5}$, but whether vaccination provides equivalent protection to natural infection in children is unknown.

In March 2021, Public Health England (PHE) initiated the SAFE-KIDS study to assess immune responses in children receiving a COVID-19 vaccine as part of the JCVI recommendations at the time. Uniquely, instead of the 3-4 week interval used by most other countries, the JCVI recommendations included a 12-week interval between COVID-19 vaccine doses, to maximise population first dose coverage. In 
adults, it is now evident that an extended interval schedule provides higher peak antibody responses after the second dose and, potentially, longer duration of protection ${ }^{6}$.

Here, we provide detailed characterisation of the antibody and cellular immune response to vaccine in fifteen children aged $12-16$ years in the United Kingdom who received either ChAdOx1 $(n=6)$ or mRNA vaccine $(n=9)$, including three donors with serological evidence of SARS-CoV-2 infection prior to vaccination. Participant characteristics are summarised in Supplementary Table 1, with details of dosing and sample timing in Supplementary Figure 1. Our study is, therefore, one of the first to determine the immunogenicity of mRNA and adenoviral-based vaccines with extended interval delivery in children.

We firstly determined antibody responses using the Meso-Scale Diagnostics (MSD) assay platform, allowing comparison with other studies using the same platform ${ }^{7,}{ }^{8}$. Samples were assessed longitudinally from baseline vaccination. All vaccines induced robust spike-specific (Figure 1A) and receptor-binding domain- (RBD-) specific (Figure 1B) antibodies. mRNA vaccine, however, induced 4.37-fold and 6.56-fold higher spike-specific antibody responses than the adenovirus-based vaccine after the first and second dose, respectively. Children who were previously naturally infected prior to vaccination demonstrated a marked increase in antibody levels after the first mRNA vaccine, spikespecific antibody levels were significantly higher than one or two doses of ChAdOx1, and significantly higher than one dose of mRNA vaccine $(p=0.001,0.004$ and 0.003 respectively, one-way ANOVA with Dunnett's multiple comparisons test), but similar to two doses of mRNA vaccine in previously uninfected children. Antibody levels after ChAdOx1 vaccine, while lower than mRNA vaccination, were 3.30-fold higher after second dose and 2.13-fold higher at three months, than those seen 2-4 months after natural infection in childhood (Supplementary Table 2). Higher antibody responses following mRNA vaccination or boosting of naturally-acquired immunity are consistent with previous studies in adults $^{7,8,9,10}$. The antibody level after mRNA vaccination were similar to those previously reported in adults $^{7}$, but were higher in children than adults after ChAdOx1 vaccination ${ }^{8}$. Waning of antibody 
responses is evident in adults ${ }^{9,}{ }^{11}$. Encouragingly, however, ChAdOx1 vaccinated children show antibody titre remains relatively stable at 3 months (Figure 1A\&B). These data are consistent with stable antibody responses between $6-12$ mths following natural infection in children ${ }^{5}$, suggesting the antibody response generated in children is uniquely durable, and is consistent with clinical protection ${ }^{4}$. Antibody levels for all cohorts are shown relative to the WHO reference standard in Supplementary Table 2.

We reported previously 'back-boosting' of seasonal coronavirus (HCoV) specific antibody levels following natural infection in children aged 3-11 years ${ }^{5}$. After the first dose of vaccine, there was a significant increase in HKU1-specific and OC43-specific antibody levels ( $p=0.0005,0.0269$ respectively, two-tailed Wilcoxon test), with no change in responses to alpha-coronaviruses (Supplementary Figure 2). This is in keeping with cross-reactivity generated in children after natural SARS-CoV- 2 infection ${ }^{5}$, or induced in adults following full vaccination?

We next assessed functional neutralisation using live wild-type (B), Alpha (B1.1.7), Beta (B1.351) or Delta (B1.617.2) viral variants. After the first dose of vaccine, neutralisation titres (NT50) were low or similar to natural infection against WT virus only in naïve donors. Neutralisation of variants was in general below detection. In contrast, previously infected donors induced high neutralising titres to all viral variants (Figure 1C). Neutralizing titres were improved after the second dose in naïve donors, including to variants (Figure 1D) although no advantage was observed with the second dose in previously-infected donors, consistent with studies in naïve and previously-infected adults. ${ }^{12}$ mRNA vaccine induced higher neutralising titres although there was considerable variability between donors. To further analyse neutralisation of viral variants, we used an MSD-ACE2 binding assay to determine the level of antibody inhibiting the interaction of ACE2 to spike from further viral variants. We found that the results from the live neutralisation assay are likely to be widely applicable to other viral variants (Supplementary Figure 3). 
Cellular immune responses are likely to be more durable than antibody responses and provide longerterm protection against severe disease ${ }^{13}$. Additionally, $\mathrm{T}$ cells are likely to be less susceptible to changes in viral variants ${ }^{14}$. As such, induction of cellular responses should be an important aim of SARSCoV-2 vaccination in children. As such we investigated the cellular immune response after two vaccine doses in three ChAdOx1 and seven mRNA vaccinated children, including two with prior infection. Spike-specific cellular responses were assessed using an activation-induced marker (AIM) assay to identify T cells responding to stimulation with a pool of overlapping-peptides from spike protein, an example is provided in Supplementary Figure 4. As previously reported in adults ${ }^{15}$, responses were dominated by CD4 T cells, with 10-fold lower CD8 T cell responses. The CD4 T cell response was higher in 4/5 mRNA vaccinated children compared to ChAdOx1 (Figure 2A). Cellular samples were also available after one vaccine dose for children receiving mRNA vaccine, allowing the trajectory of $\mathrm{T}$ cell responses to be assessed. The T cell response peaked after one dose in previously-infected children, consistent with results from adults ${ }^{12}$. In contrast, the CD4 T cell response increased significantly $(p=0.0470$, two-tailed paired t-test) following second dose in infection-naïve donors (Supplementary Figure 5A).

As such, mRNA vaccines generate particularly robust spike-specific cellular responses in children, which contrasts with previous reports in adults where ChAdOx1 induced somewhat higher cellular responses ${ }^{7,8,16}$. Overall, these findings show that mRNA vaccination induces superior coordinated cellular and antibody responses in children compared to adenovirus-based vaccines within the timescale of this investigation.

We next characterised the CD4 response in more detail. In all vaccine types the predominant response was early $C D 27+C D 28+$ effector memory, with elements of central memory, indicating induction of a memory response (Figure 2B; Supplementary Figure 5B). CXCR3 and CCR4 expression characterise Th1 and Th2 CD4+ populations, respectively, and both were represented within the spike-specific 
response at similar frequencies. CXCR5+ T follicular helper cells were also present within the antigenspecific pool (Figure 2C; Supplementary Figure 5C).

The cytokine profile of spike-specific T cells was determined by analysis of supernatant from AIM assay cultures. Type-I cytokines were found at high levels, with IFNY and IL-2 predominating; release of TNF was also notable, consistent with a Type-I cytokine profile. The level of IFN $\gamma$ was significantly higher in mRNA vaccinated donors than ChAdOx1 $(p=0.0155$, RM two-way ANOVA with GeisserGreenhouse correction and Tukey's multiple comparison test). Cells also demonstrated cytotoxic potential, with release of perforin, granzyme A and B. The response showed little evidence of IL-4 production, indicating the high proportion of activated CCR4-expressing cells are unlikely to be Th2polarised cells. Rather, the presence of spike-specific T cells expressing CCR4 in the absence of Th2 cytokines suggests a proportion of the induced T cells are primed for lung homing ${ }^{17}$. IL-17A was also below $10 \mathrm{pg} / \mathrm{ml}$. However, high IL-10 concentrations were found within the supernatant and this is noteworthy as its coordinated production with Type-I cytokines has been observed as a feature of mild and asymptomatic infection (Figure 2D) $)^{5,15,18}$. The profile of cytokine release did not change between first and second dose in mRNA vaccinated children (Supplementary Figure 5D) and was consistent between naïve and previously-infected children. Thus, these data show mRNA vaccination induces a coordinated robust cellular immune response in children.

Finally, to assess the relationship between the antibody response and cellular response, we performed correlation analysis. Significant correlations between total spike-specific antibody response and neutralising antibody titre were present. Likewise, there was a significant correlation between total CD4 T cell response and neutralising antibody levels, indicating a highly-coordinated cellular and antibody response. There was also a significant correlation between anti-RBD antibody titres and CCR4+ spike-specific T cells, with a positive correlation between CCR4+ cells and neutralising and spike antibody levels also present. CCR4+ CD4 T cells have been associated with influenza vaccine response 13. A trend towards a negative correlation between antibody response and Tfh and CXCR3+ Tfh cells 
was also seen (Supplementary Figure 6), which contrasts with a positive correlation in adults ${ }^{19}$. This may suggest either differential regulation of antibody production in children or increased recruitment of Th cells into lymphoid tissue.

The UK JCVI decision to recommend COVID-19 vaccination for children with severe neuro-disabilities soon after COVID-19 vaccines were authorised for adults provided a unique opportunity to rapidly assess immune responses to two different types of vaccines in a real-world setting. There are very limited data published on immune responses to COVID-19 vaccination in children and none comparing responses in children who received ChAdOx1 against an mRNA vaccine ${ }^{2,3,4}$. Our finding of higher immunogenicity with mRNA vaccine compared to ChAdOx1 vaccine is consistent with data in adults ${ }^{7}$ $8,9,10$, including studies reporting the extended 12-week interval schedule, which elicits higher peak antibody levels and longer duration of protection after the second dose than 3-4-week interval schedule ${ }^{6,7}$. Overall, our findings would support use of an extended mRNA vaccination schedule in children, instead of the 3-4-week interval for mRNA vaccines.

A limitation of this study is the small number of participants, mainly due to very low vaccine uptake in eligible groups during the first 6 months of 2021. Nationally, only 750 children were vaccinated, despite thousands of high-risk children being eligible for vaccination ${ }^{20}$. While regarded as higher risk, our participants were not immunosuppressed and, as such, should be representative of immunity in the general adolescent population. Consistently, antibody responses in children infected prior to vaccination in our cohort were similar to naturally-infected healthy children. Our cohort of naturallyinfected children were likely to have been infected to the original SARS-CoV-2 strain and, as such, had similar exposure to the vaccine strain spike sequence, providing a useful threshold to compare the level and breadth of immune responses after vaccination in children.

Our study provides a unique, detailed, insight into the relative immunogenicity of mRNA or adenoviralvector vaccines in adolescents and indicates mRNA vaccines provide stronger immune responses following the second vaccine dose. The data also provide support for the use of two mRNA doses for 
seronegative children. The high response achieved in previously naturally-infected children indicates that a single dose is likely to be sufficient in these children. 

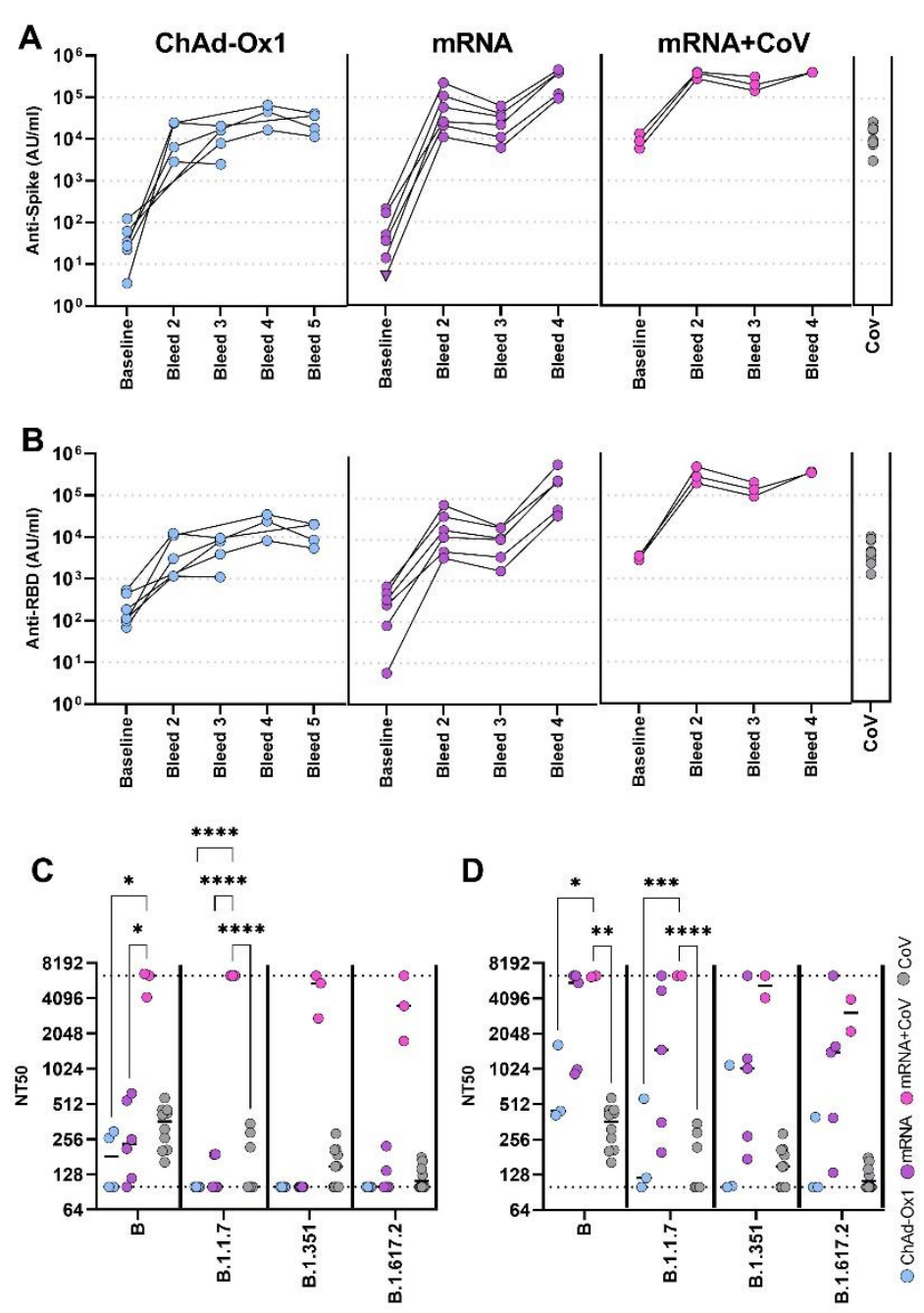

\section{Figure 1. Antibody responses in children following CoVID-19 vaccination.}

Antibody levels to Spike (A) and RBD (B) measured by MSD assay in children receiving CoVID-19 vaccination (ChAdOx1 - seronegative children receiving ChAdOx1 vaccination, $(n=6)$, mRNA seronegative children receiving mRNA vaccination $(n=6), m R N A+C o V-$ seropositive children receiving mRNA vaccination $(n=3)$ ). Antibody levels $2-4$ months following natural CoVID-19 infection $(\mathrm{CoV},(\mathrm{n}=10))$ are shown for comparison. C\&D) Neutralisation of live virus, either wild type B (PHE-2), B1.1.7 (alpha), B1.351 (beta) or B1.617.2 (delta) variants, following first dose (C) or following second dose (D). Dotted lines represent upper and lower limits of detection. RM Two-way ANOVA with Geisser-Greenhouse correction and Tukey's multiple comparison test. 

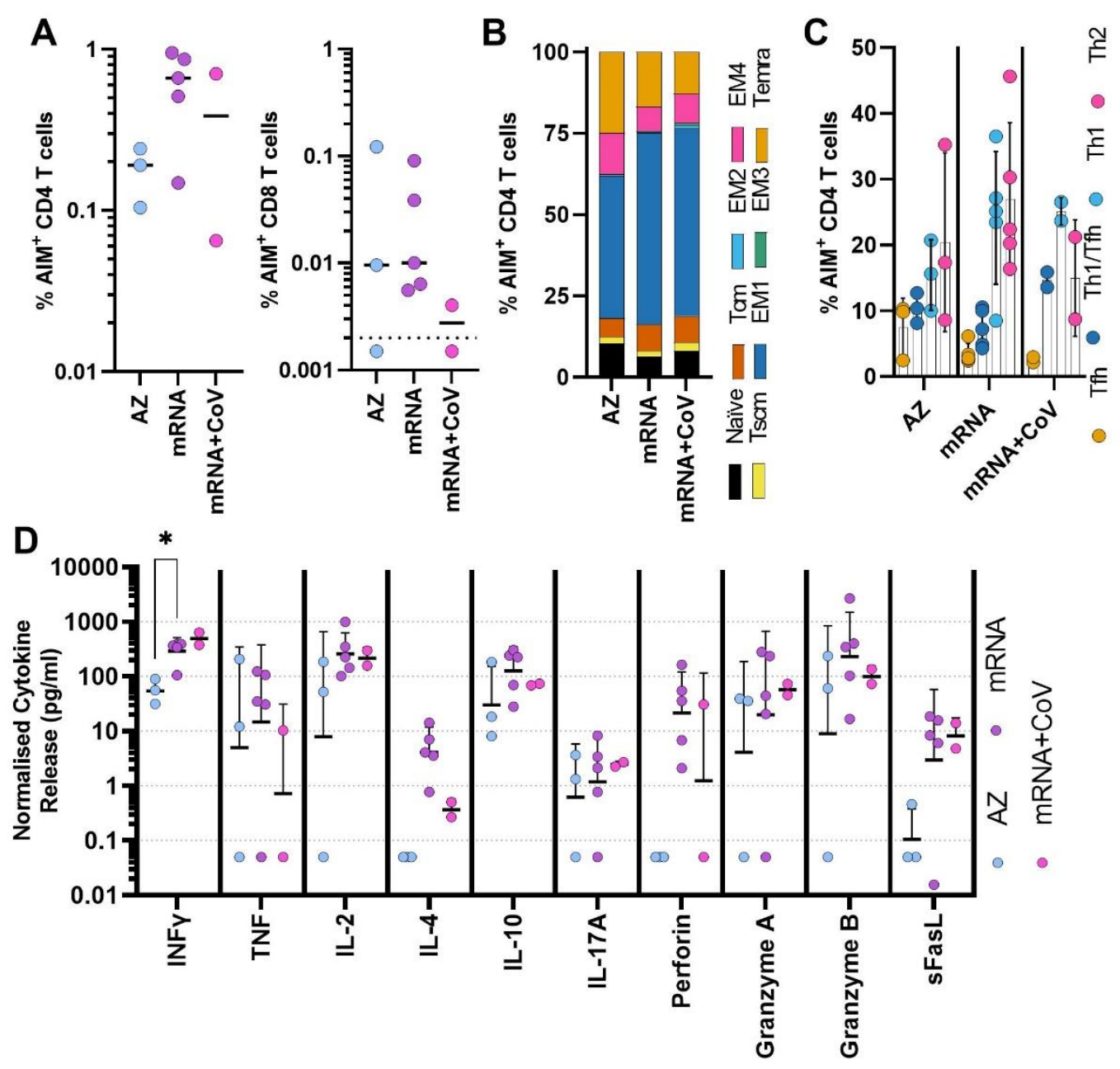

Figure 2. Cellular responses in children following CoVID-19 vaccination. Analysis of the T cell response following the second dose of vaccine. A flow cytometry based AIM assay was used to identify responding CD4 (CD69+CD40L+) and CD8 (CD69+CD137+) T cell frequency following overnight stimulation with overlapping spike peptides $(A)$, dots indicate individual donors, bars indicate median. Aim + CD4 T cells, were phenotyped to assess memory state (B) bars represent proportion of the Aim $+C D 4$ T cell population, data is mean of $n=3 A Z, n=5$ mRNA and $n=2$ mRNA+CoV. Teffector memory (CD45ra-CCR7-, EM) are subdivided as EM1 - CD27+CD28+, EM2 CD27+CD28-, EM3 - CD27-CD28-, EM4-CD27-CD28+). C) the expression of polarisation markers CXCR5 (Tfh) CXCR3 (Th1) and CCR4 (Th2), dots indicate individual donors, bars indicate mean \pm SD. D) Supernatant from overnight stimulated cultures were analysed to identify cytokine production. Data was normalised to $1 \times 10^{6} \mathrm{PBMC}$ per well, cytokine release is shown minus background cytokine production in unstimulated (DMSO) wells. Dots indicate individual donors, bars indicate geometric mean \pm geo.SD. Repeated measure two-way ANOVA with Geisser-Greenhouse correction and Tukey multiple comparisons test. 
Supplementary Table 1. Demographics and Vaccination details

\begin{tabular}{|c|c|c|c|c|}
\hline Cohort & ChAdOx1 & mRNA & mRNA+CoV & CoV \\
\hline Donors & $n=6$ & $n=6$ & $\mathrm{n}=3$ & $\mathrm{n}=10$ \\
\hline Age & $13(12-16)$ & $14(13-15)$ & $14(13-15)$ & $11(10-13)$ \\
\hline $\begin{array}{l}\text { Gender (Male: } \\
\text { Female) }\end{array}$ & $2: 1$ & $2: 1$ & $2: 1$ & $3: 2$ \\
\hline Vaccine & $\begin{array}{l}\text { ChAdOx1 nCoV- } \\
19\end{array}$ & $\begin{array}{l}\text { mRNA-1273 } \\
(n=5), \\
\text { BNT162b2 }(n=1)\end{array}$ & $\begin{array}{l}\text { mRNA-1273 } \\
(n=3)\end{array}$ & - \\
\hline $\begin{array}{l}\text { Dose two } \\
\text { Interval }\end{array}$ & $\begin{array}{l}10.57 \text { weeks } \\
(10.14-10.57)\end{array}$ & $\begin{array}{l}11.43 \text { weeks } \\
(9.00-13.14)\end{array}$ & $\begin{array}{l}11.43 \text { weeks } \\
(11.43-16.29)\end{array}$ & - \\
\hline Time from PCR & - & - & - & $\begin{array}{l}18.08 \text { weeks } \\
(12.40-21.40) \\
\end{array}$ \\
\hline \multicolumn{5}{|l|}{ Comorbidities } \\
\hline Cerebral palsy & $\mathrm{n}=2$ & $n=3$ & - & - \\
\hline $\begin{array}{l}\text { Congenital } \\
\text { muscular } \\
\text { dystrophy }\end{array}$ & $n=1$ & - & $n=1$ & - \\
\hline $\begin{array}{l}\text { Congenital } \\
\text { metabolic } \\
\text { disorder }\end{array}$ & $n=1$ & - & - & - \\
\hline $\begin{array}{l}\text { Down syndrome } \\
\text { with neurological } \\
\text { complications }\end{array}$ & $n=1$ & - & - & - \\
\hline $\begin{array}{l}\text { Epilepsy with } \\
\text { severe learning } \\
\text { difficulties }\end{array}$ & $n=1$ & $n=5$ & $n=2$ & - \\
\hline $\begin{array}{l}\text { Alpha- } \\
\text { thalassemia x- } \\
\text { linked } \\
\text { intellectual } \\
\text { disability (ATRX) } \\
\text { syndrome }\end{array}$ & - & $\mathrm{n}=1$ & - & - \\
\hline
\end{tabular}




\begin{tabular}{|c|c|c|c|c|c|}
\hline & Vaccine type & $\begin{array}{l}\text { Spike AU/ml } \\
\text { ( } \pm \text { SD) }\end{array}$ & $\begin{array}{l}\text { RBD AU/ml } \\
( \pm S D)\end{array}$ & $\begin{array}{l}\text { Spike BAU/ml } \\
\text { ( } \pm \text { SD) }\end{array}$ & $\begin{array}{l}\text { RBD BAU/ml } \\
\text { ( } \pm \text { SD) }\end{array}$ \\
\hline \multirow{3}{*}{ 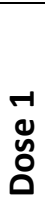 } & ChAdOx1 & $10259 \pm 2.88$ & $4695 \pm 3.06$ & $92.43 \pm 0.03$ & $127.7 \pm 0.08$ \\
\hline & mRNA & $44856 \pm 3.05$ & $14497 \pm 3.11$ & $404.15 \pm 0.03$ & $394.31 \pm 0.08$ \\
\hline & mRNA+CoV & $352762 \pm 1.22$ & $300969 \pm 1.58$ & $3178.38 \pm 0.01$ & $8186.35 \pm 0.04$ \\
\hline \multirow{3}{*}{ 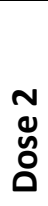 } & ChAdOx1 & $36519 \pm 2.03$ & $19070 \pm 2.11$ & $329.03 \pm 0.02$ & $518.7 \pm 0.06$ \\
\hline & mRNA & $239519 \pm 2.12$ & $156116 \pm 3.34$ & $2158.06 \pm 0.02$ & $4246.35 \pm 0.09$ \\
\hline & mRNA+CoV & $396470 \pm 1.01$ & $359518 \pm 1.04$ & $3572.19 \pm 0.01$ & $9778.89 \pm 0.03$ \\
\hline \multirow{4}{*}{ 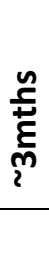 } & ChAdOx1 & $23516 \pm 1.83$ & $11764 \pm 1.93$ & $211.88 \pm 0.02$ & $319.98 \pm 0.05$ \\
\hline & mRNA & - & - & - & - \\
\hline & mRNA+CoV & - & - & - & - \\
\hline & CoV & $11059 \pm 1.88$ & $4398 \pm 1.98$ & $99.64 \pm 0.02$ & $119.63 \pm 0.05$ \\
\hline
\end{tabular}

Supplementary Table 2. Antibody titres following vaccination. Shown are Geo. Mean titres for Spike and RBD for each group as indicated following first or second dose, as shown in Figure $1 \mathrm{~A}$ and 1B. Data are presented as $\mathrm{AU} / \mathrm{ml}$, data are also provided as $\mathrm{BAU} / \mathrm{ml}$ compatible with the WHO reference standard. 


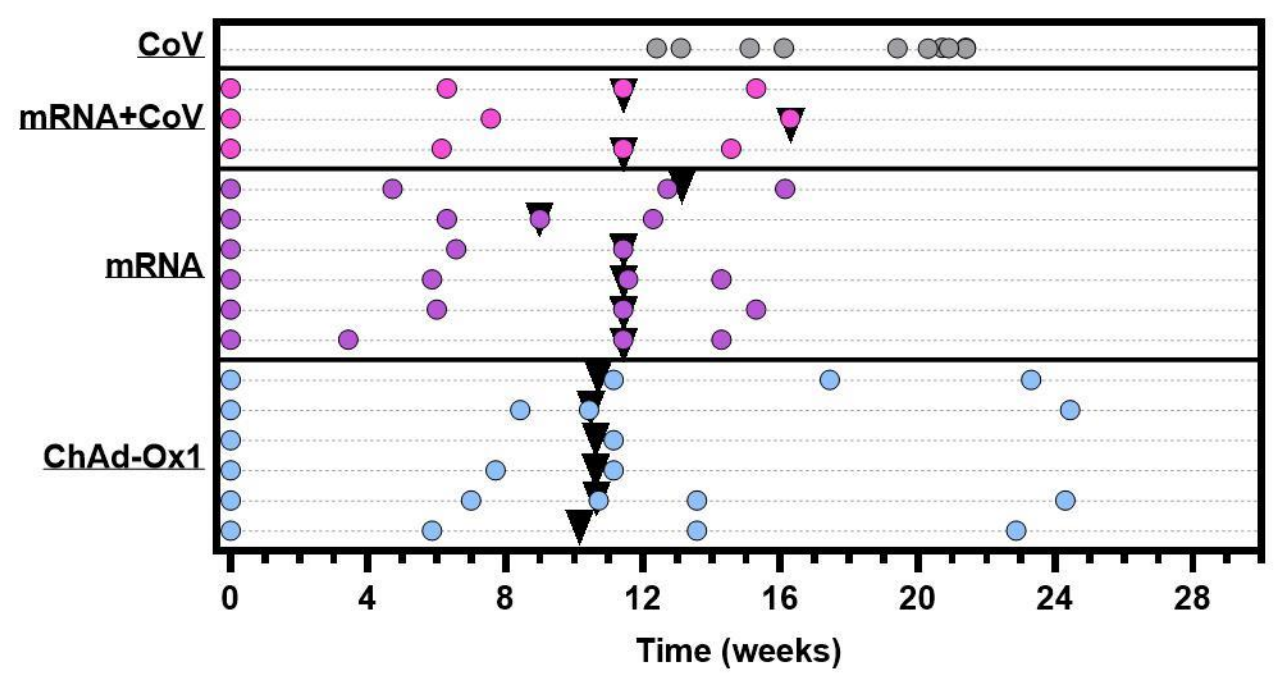

Supplementary Figure 1. A graphical representation of sample collection and vaccine administration. Vaccinated.

Children receiving CoVID-19 vaccination (mRNA+CoV - seropositive children receiving mRNA vaccination $(n=3)$, mRNA - seronegative children receiving mRNA vaccination $(n=6)$, ChAdOx1 seronegative children receiving ChAdOx1 vaccination $(n=6))$ are shown on separate rows for individual donors, dots represent sample collection for serology or serology and cellular analysis. Naturally infected children with definitive PCR results are shown together ( $\mathrm{CoV}, \mathrm{n}=10$ ). Black triangles indicate time of second dose. Time is relative to administration of first dose, or date of PCR. 

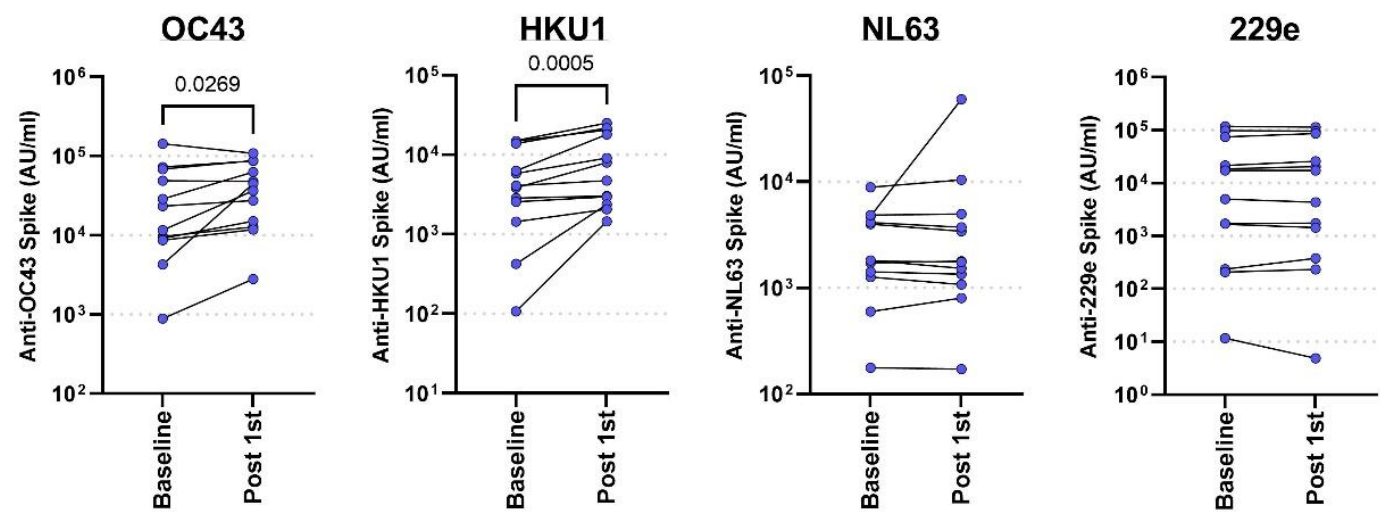

Supplementary Figure 2. HCoV antibody levels following a single dose of CoVID-19 vaccine in seronegative children.

Antibody levels to the beta-coronavirus - OC43 and HKU1 and the alpha-coronavirus NL63 and 229e were measured by MSD assay in seronegative children receiving either ChAdOx1 or mRNA vaccine $(n=12)$ at baseline and following first dose of vaccine. Dots represent individual donors, lines connect timepoints. Two-tailed Wilcoxon t-test. 

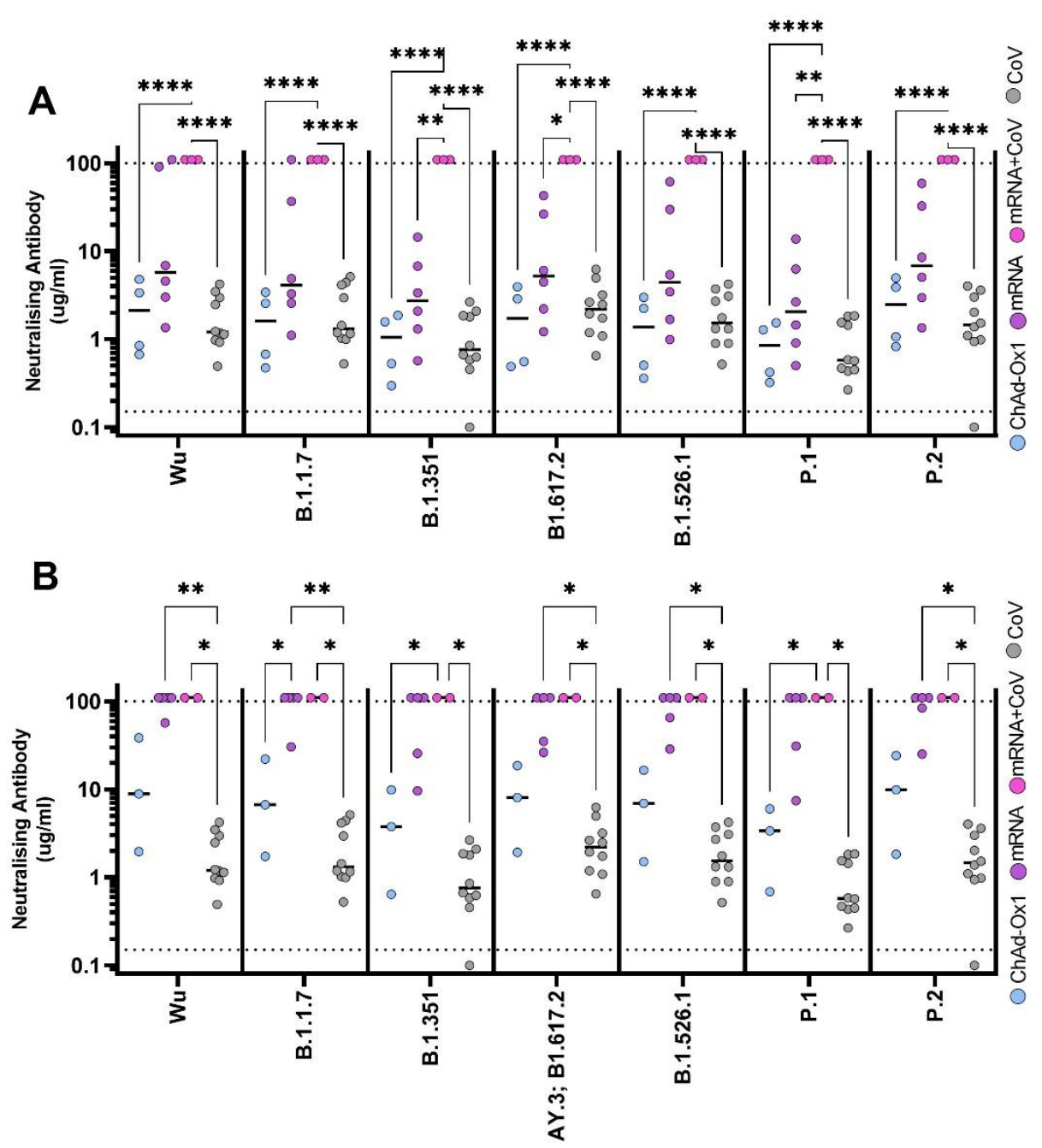

Supplementary Figure 3. Neutralising antibody levels from Wuhan (Wu) Spike or Spike from variants of concern. The level of neutralising antibody was determined by pseudo-neutralisation of Spike-ACE2 binding using MSD following first (A) or second (B) dose of vaccine. (A) ChAdOx1 seronegative children receiving ChAdOx1 vaccination, $(n=4)$, mRNA - seronegative children receiving mRNA vaccination $(n=6), m R N A+C o V-$ seropositive children receiving mRNA vaccination $(n=3))$. Antibody levels following natural CoVID-19 infection (CoV, $(n=10)$ ) are shown for comparison. (B) ChAdOx1 - seronegative children receiving ChAdOx1 vaccination, $(n=3)$, mRNA - seronegative children receiving $m R N A$ vaccination $(n=5), m R N A+C o V$ - seropositive children receiving mRNA vaccination $(n=2))$. Antibody levels following natural CoVID-19 infection (CoV, $(n=10))$ are shown for comparison. Mixed-effect model with Geisser-Greenhouse correction and Tukey multiple comparisons test. 

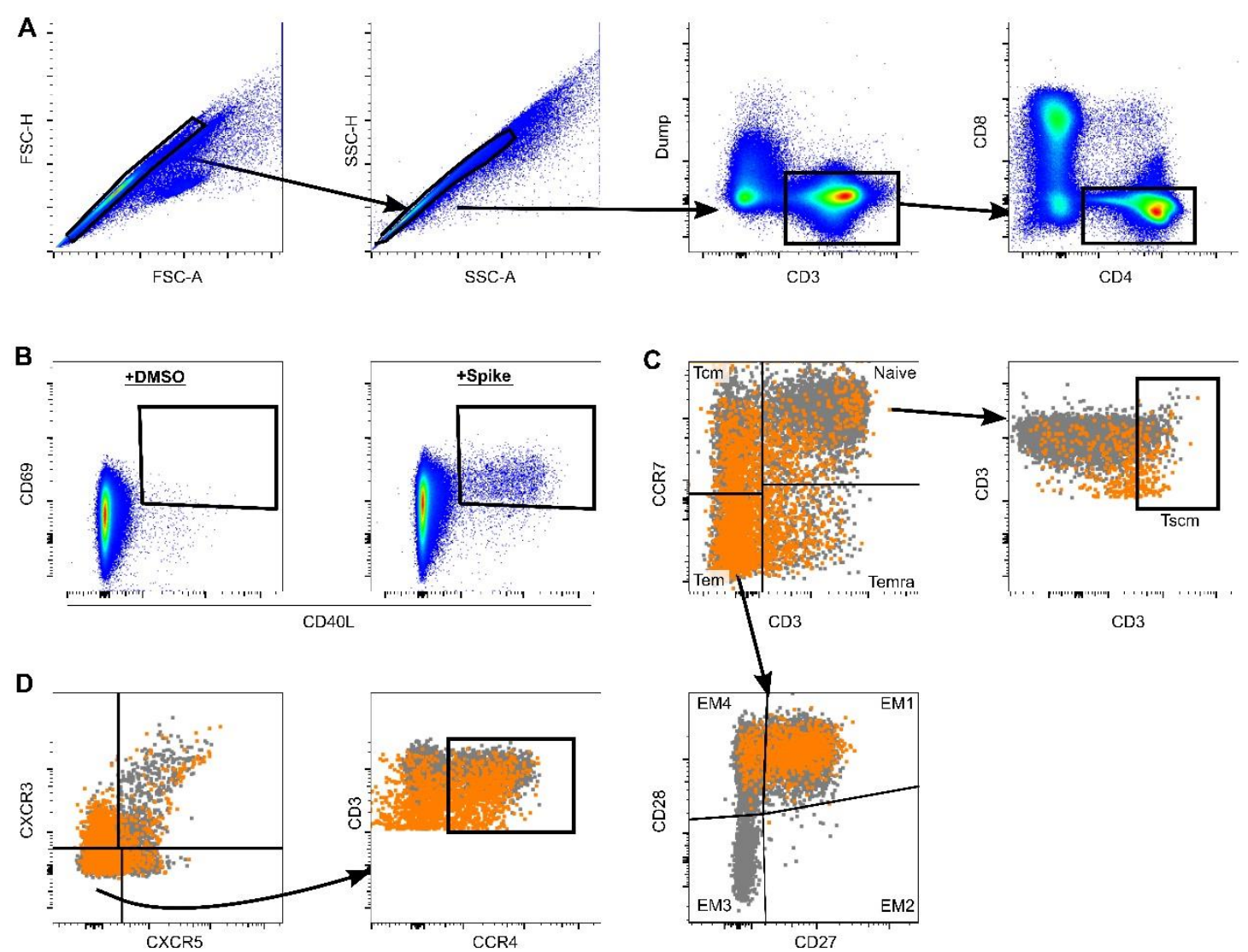

Supplementary Figure 4. Gating strategy for analysis of cellular responses. A) shows the initial gating strategy to identify CD4 T cells as shown. B) shows an example of AIM assay staining from a mRNA vaccinated child, showing negative control (+DMSO) and cultures stimulated with overlapping spike peptides. C\&D) show examples of further gating and staining from the same donor, the AIM positive population is shown (orange) and the AIM negative population (grey). C) Shows assessment of memory populations, Naïve (CD45ra+CCR7+) population were sub-gated to identify Tscm (CD45ra+CCR7+CD95+), likewise the Tem (CD45ra-CCR7-) population was sub-gated and EM1-4 populations defined by CD27/CD28 expression. D) CXCR3 and CXCR5 populations were first identified, and CCR4 expression assessed on the CXCR3-CXCR5- population. 

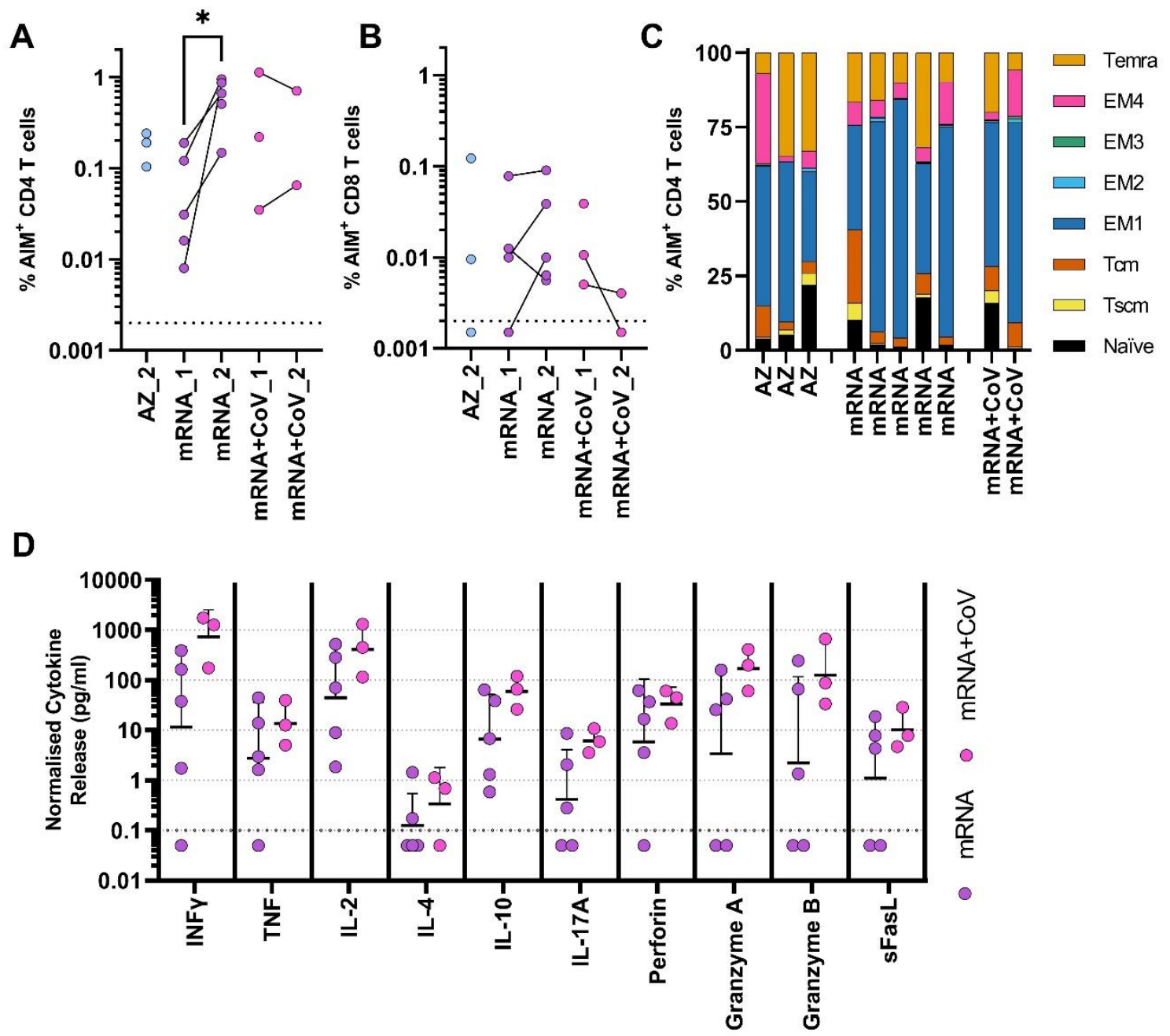

Supplementary Figure 5. Cellular responses in children following CoVID-19 vaccination. A flow cytometry based AIM assay was used to identify responding A) CD4 (CD69+CD40L+) and B) CD8 (CD69+CD137+) T cell frequency following overnight stimulation with overlapping spike peptides. T cell response in mRNA vaccinated children following first dose of vaccine (mRNA_1, mRNA+CoV_1) are shown. Results following the second dose of vaccine are also shown for comparison (AZ_2, mRNA_2, mRNA+CoV_2). Dots indicate individual donors, lines connect timepoints. Two-tailed paired t-test. Aim + CD4 T cells, were phenotyped to assess memory state (C), columns show individual donors, bars represent proportion of the Aim $+C D 4$ T cell population, $n=3 \quad A Z, n=5$ mRNA and $n=2$ mRNA+CoV. D) Supernatant from overnight stimulated cultures of PBMC from mRNA vaccinated children following first vaccine were analysed to identify cytokine production. Data was normalised to $1 \times 10^{6} \mathrm{PBMC}$ per well, cytokine release is shown minus background cytokine production in unstimulated (DMSO) wells. Dots indicate individual donors, bars indicate geometric mean \pm geo.SD. 

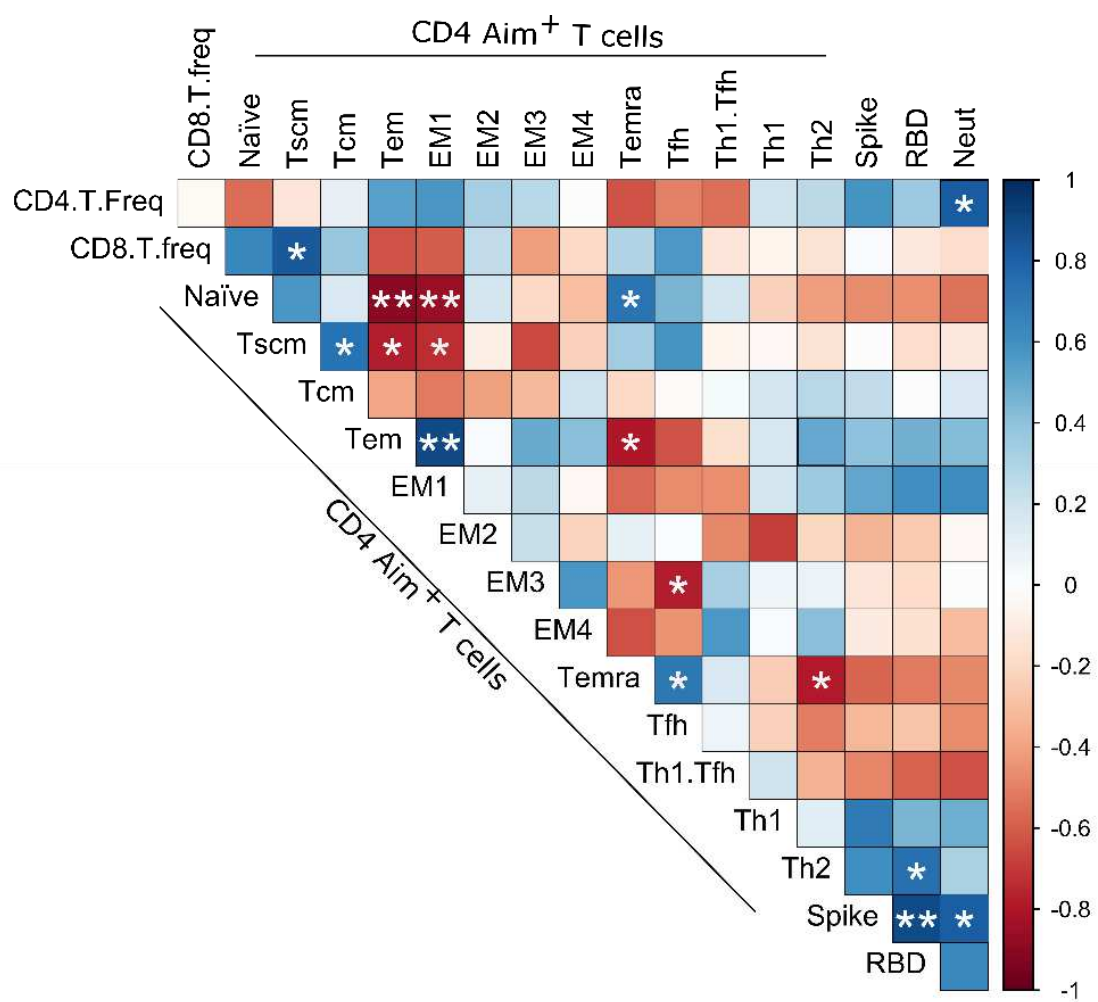

Supplementary Figure 6. Correlation plot of measured variables following second vaccine.

Variables from cellular and serology assays from previously seronegative children vaccinated with either ChAdOx1 or mRNA vaccine, were correlated using the R-corrplot package. Degree of correlation is indicated by strength of colour; positive correlation blue, negative correlation red. Significantly correlated results are indicated by ${ }^{*} p<0.05,{ }^{* *} p<0.01$. 


\section{References}

1. Wong, B.L.H., Ramsay, M.E. \& Ladhani, S.N. Should children be vaccinated against COVID-19 now? , archdischild-2020-321225 (2021).

2. Ali, K. et al. Evaluation of mRNA-1273 SARS-CoV-2 Vaccine in Adolescents. (2021).

3. Frenck, R.W. et al. Safety, Immunogenicity, and Efficacy of the BNT162b2 Covid-19 Vaccine in Adolescents. 385, 239-250 (2021).

4. Tartof, S.Y. et al. Effectiveness of mRNA BNT162b2 COVID-19 vaccine up to 6 months in a large integrated health system in the USA: a retrospective cohort study. The Lancet 398, 1407-1416 (2021).

5. Dowell, A.C. et al. Children develop robust and sustained cross-reactive spike-specific immune responses following SARS-CoV-2 infection. 2021.2004.2012.21255275 (2021).

6. Parry, H. et al. Extended interval BNT162b2 vaccination enhances peak antibody generation in older people. 2021.2005.2015.21257017 (2021).

7. Payne, R.P. et al. Immunogenicity of standard and extended dosing intervals of BNT162b2 mRNA vaccine. Cell (2021).

8. Folegatti, P.M. et al. Safety and immunogenicity of the ChAdOx1 nCoV-19 vaccine against SARS-CoV-2: a preliminary report of a phase $1 / 2$, single-blind, randomised controlled trial. The Lancet 396, 467-478 (2020).

9. Shrotri, M. et al. Spike-antibody waning after second dose of BNT162b2 or ChAdOx1. The Lancet 398, 385-387 (2021).

10. Sadarangani, M., Marchant, A. \& Kollmann, T.R. Immunological mechanisms of vaccineinduced protection against COVID-19 in humans. Nature Reviews Immunology 21, 475-484 (2021).

11. Pegu, A. et al. Durability of mRNA-1273 vaccine\&\#x2013; induced antibodies against SARSCoV-2 variants. 373, 1372-1377 (2021).

12. Mazzoni, A. et al. First-dose mRNA vaccination is sufficient to reactivate immunological memory to SARS-CoV-2 in subjects who have recovered from COVID-19. The Journal of Clinical Investigation 131 (2021). 
13. Pallikkuth, S. et al. Dysfunctional peripheral T follicular helper cells dominate in people with impaired influenza vaccine responses: Results from the FLORAH study. PLOS Biology 17, e3000257 (2019).

14. Skelly, D.T. et al. Two doses of SARS-CoV-2 vaccination induce robust immune responses to emerging SARS-CoV-2 variants of concern. Nature Communications 12, 5061 (2021).

15. Zuo, J. et al. Robust SARS-CoV-2-specific T-cell immunity is maintained at 6 months following primary infection. Nat Immunol 22, 620-626 (2020).

16. Parry, H. et al. Differential immunogenicity of BNT162b2 or ChAdOx1 vaccines after extended-interval homologous dual vaccination in older people. Immunity \& Ageing 18, 34 (2021).

17. Mikhak, Z., Strassner, J.P. \& Luster, A.D. Lung dendritic cells imprint T cell lung homing and promote lung immunity through the chemokine receptor CCR4. Journal of Experimental Medicine 210, 1855-1869 (2013).

18. Le Bert, N. et al. Highly functional virus-specific cellular immune response in asymptomatic SARS-CoV-2 infectionSARS-CoV-2-specific T cells in asymptomatic. Journal of Experimental Medicine 218 (2021).

19. Painter, M.M. et al. Rapid induction of antigen-specific CD4+ T cells is associated with coordinated humoral and cellular immunity to SARS-CoV-2 mRNA vaccination. Immunity 54, 2133-2142.e2133 (2021).

20. Aiano, F., Campbell, C., Saliba, V., Ramsay, M.E. \& Ladhani, S.N. COVID-19 vaccine given to children with comorbidities in England, December 2020-June 2021. archdischild-2021323162 (2021). 


\section{Supplementary Files}

This is a list of supplementary files associated with this preprint. Click to download.

- SAFEKidsSupMethod.pdf 